\title{
Retinal Dystrophy, Obesity and Hypertension in an Adolescent Boy
}

\author{
Rabia Ali ${ }^{1}$, Khalid Kamal ${ }^{2}$, and Sikandar Saeed ${ }^{3}$ \\ ${ }^{1}$ Affiliation not available \\ ${ }^{2}$ Children's Hospital of Michigan \\ ${ }^{3}$ Shifa College of Medicine
}

November 9, 2020

\begin{abstract}
Bardet-Biedl syndrome (BBS) is a pleiotropic genetic disorder characterized by congenital and late onset defects. It is associated with retinal dystrophy, obesity, polydactyly, hypogonadism and renal dysfunction. Proper management and regular health visits are necessary to avoid complications. We present a patient with retinal dystrophy, obesity and new onset hypertension.
\end{abstract}

\section{Retinal Dystrophy, Obesity and Hypertension in an Adolescent Boy}

Rabia $\mathrm{A}^{\mathrm{a}}$, Khalid $\mathrm{K}^{\mathrm{b}}$, MD, Sikandar $\mathrm{S}^{\mathrm{a}}$.

Affiliations: ${ }^{a}$ Shifa College of Medicine, Shifa Tameer-E-Millat University, Islamabad, Pakistan, and ${ }^{\mathrm{b}}$ Children's Hospital of Michigan, Detroit, MI, USA.

Address Correspondence to: Rabia Ali, Department of Medicine, Shifa College of Medicine, NCBMS Tower, Sector H-8/4, service road S, near Federal Board Office, H 8/4 H-8, Islamabad, Islamabad Capital Territory 46000, [rabiaali5266@gmail.com], +92 3129441869

Short title: A case of retinal dystrophy, obesity and hypertension

Funding Source: No funding was secured for this study

Financial Disclosure: The authors do not have any financial relationships relevant to this article to disclose.

Conflict of Interest: The authors have no conflict of interest to disclose.

Acknowledgement: none

\section{Abbreviations:}

BBS: Bardet Biedl Syndrome

ACE: Angiotensin Converting EnzymeAbstractBardet-Biedl syndrome (BBS) is a pleiotropic genetic disorder characterized by congenital and late onset defects. It is associated with retinal dystrophy, obesity, polydactyly, hypogonadism and renal dysfunction. Proper management and regular health visits are necessary to avoid complications. We present a patient with retinal dystrophy, obesity and new onset hypertension.Key words : Bardet-Biedl syndrome, congenital, adolescence, obesity, retinal dystrophy, hypertension.IntroductionBardet Biedl Syndrome (BBS) is a pleiotropic genetic disorder with an autosomal recessive mode of inheritance. Laurence and Moon, two ophthalmologists, first reported it in 1866. BBS occurs because of a defect in BBS genes that code for many ciliary-related proteins. Therefore, it is classified as a ciliopathic disorder that arises due to ciliary dysgenesis and dysfunction ${ }^{[1]}$. To this date, 20 genes have been identified and all of them disrupt ciliary function. The prevalence of BBS varies from 1:160,000 
in Northern European populations to $1: 13,500$ and 1:17,500 in Kuwait and Newfoundland respectively ${ }^{[2]}$. Genetic testing confirms the diagnosis in most cases. However, diagnosis is primarily clinical ${ }^{[3]}$.

According to the Bardet Biedl Syndrome (BBS) modified diagnostic criteria proposed by Beales et al, our patient met 5 of 6 primary clinical features (rod-cone dystrophy, polydactyly, obesity, learning difficulties, hypogonadism). Differential diagnosis of BBS includes Laurence-Moon syndrome, Alström Syndrome and Prader-Wili Syndrome. Laurence-Moon syndrome exhibits spastic paraparesis and distal muscle weakness whereas polydactyly is seen in BBS.

\section{Case Presentation}

A 17-year old boy presents to the pediatric clinic for an annual interim evaluation. He weighs $82.3 \mathrm{~kg}$ and his height is $1.76 \mathrm{~m}$. His body mass index is 26.4 , which is above the $85^{\text {th }}$ percentile, in the overweight category. Current complaints include hypertension, scoliosis and vision difficulties. He is otherwise alert with no signs of distress and has no complaints of pain.

He has had progressively deteriorating eyesight since he was born, and was prescribed magnifying glasses. His visual acuity is $20 / 30$ in both eyes. He sees without difficulty during the day but has night blindness. Rodcone dystrophy was previously confirmed by electroretinography. He has also developed hypertension over the past year. Blood pressure is $148 / 80 \mathrm{mmHg}, 95^{\text {th }}$ percentile for systolic and $43^{\text {rd }}$ percentile for diastolic based on August 2017 American Academy of Pediatrics Clinical Guidelines.

The patient was born with polydactyly; there was a $6^{\text {th }}$ digit on both feet. These digits were surgically removed in 2012. He is flat footed and wears ankle foot orthoses for increased stability while walking. He had undescended testes, for which bilateral orchiopexy was performed before the age of one. He had obvious developmental delays throughout childhood. He receives two hours of special education alongside his high school education. The patient has a history of hydronephrosis previously, which is not found on renal ultrasonography during this visit. He has had scoliosis in his lumbar region for the past year, which does not cause any numbness, tingling or back pain. Past history reveals enlarged ventricles, macrocephaly on magnetic resonance imaging during childhood in 2010. He has regular follow-ups with his neurosurgeon and there is no history of headaches, nausea or vomiting. A five-generation pedigree, shown in Figure $\mathbf{1}$, has been obtained and significant data has been noted.

We recommended our patient to receive genetic testing and prescribed him a tablet of Lisinopril $5 \mathrm{mg}$ per oral once daily. He is referred to Ophthalmology for an annual visit and asked to return after a month. Scoliosis is being carefully monitored for any progression. On follow up, the patient had an improved blood pressure of $122 / 76 \mathrm{mmHg}$, in the $66^{\text {th }} / 80^{\text {th }}$ percentile.

\section{Discussion}

Clinical diagnosis of BBS is based on a set of modified diagnostic criteria established by Beales J. et al . According to these criteria, either four primary features or three primary and two secondary features are required to diagnose the disease ${ }^{[4]}$. Retinitis pigmentosa, polydactyly and obesity appear to be the hallmark clinical presentation ${ }^{[5]}$.

Symptoms characterized as primary and secondary features are shown inTable $\mathbf{1}$. The initial clinical presentation occurs in the first decade of life with polydactyly present at birth. The age of diagnosis varies and depends on the age at which the onset of symptomatic rod-cone dystrophy occurs. This presents as night blindness, photophobia and loss of color vision. Most patients become legally blind by the third decade of life. Electroretinography is the gold standard investigation, and may show early changes in the first two years of life. Obesity is one of the most common findings that is reported. The incidence of truncal obesity in BBS patients is reported to be $72-86 \%{ }^{[6]}$.

Renal and urinary tract anomalies and renal dysfunction; causes of considerable morbidity, are reported to affect $53 \%-82 \%$ of patients with BBS ${ }^{[7]}$. Hypertension may occur in $50 \%-60 \%$ of cases ${ }^{[8]}$. Renal disease can also be secondary to hypertension and diabetes; two conditions frequently seen in this population. A study 
carried out in 2017 suggests that patients with BBS either develop chronic kidney disease in childhood or remain entirely free of it. The proportion that does develop adult onset renal disease may also have comorbidities associated to BBS such as hypertension and diabetes. Thirty-five percent of patients were hypertensive and the most commonly prescribed antihypertensives were angiotensin converting enzyme (ACE) inhibitors [9].

At present, patients with BBS can only be treated symptomatically. The primary focus is on the management of diabetes, hypertension and metabolic syndrome in order to reduce their effect on already affected organs such as the eyes and kidneys. Weight management is a constant struggle for most patients. Many opt to have bariatric surgery or use anti-obesity medications but the safest and most effective weight loss strategy is dietary restriction ${ }^{[10]}$. As it stands, there is no definitive treatment to prevent deterioration of vision. Early evaluation by a specialist can help limit disability by providing patients with vision aids and mobility training ${ }^{[11]}$. Surgical intervention is indicated for some of the associated physical conditions, including polydactyly, some genitourinary abnormalities and congenital heart defects. In the setting of severe kidney failure later in life, kidney transplantation may be advised.

Blood pressure should be measured six monthly or more often in the setting of hypertension. Treatment of hypertension in BBS is no different from that of essential hypertension. Lifestyle modifications should be implemented to prevent hypertension. Antihypertensives and lipid-lowering medication should be prescribed as appropriate. In children and adolescents, pharmacologic treatment should be started with ACE inhibitors, angiotensin receptor blockers, long acting calcium channel blockers or thiazide diuretics ${ }^{[5]}{ }^{[12]}$.

\section{Key Clinical Message}

* Diagnosis is based on the modified diagnostic criteria proposed by Beales et al.

* Secondary conditions can start due to the presence of BBS.

* Patient needs to be carefully monitored by a multidisciplinary team for disease progression.

\section{Authorship}

Author 1: Rabia Ali conceptualized and drafted the manuscript.

Author 2: Dr Khalid Kamal added critically important information, reviewed the manuscript and added important intellectual content.

Author 3: Sikandar Saeed drafted and revised the manuscript multiple times till its final version.

All authors approved the final manuscript as submitted and agree to be accountable for all aspects of the work.

\section{References}

1. Priya S, Nampoothiri S, Sen P, Sripriya S. Bardet-Biedl syndrome: Genetics, molecular pathophysiology, and disease management. Indian J Ophthalmol . 2016;64(9):620. doi:10.4103/0301-4738.194328

2. Weihbrecht K, Goar WA, Pak T, et al. Keeping an Eye on Bardet-Biedl Syndrome: A Comprehensive Review of the Role of Bardet-Biedl Syndrome Genes in the Eye. Med Res Arch. 2017;5(9):10.18103/mra.v5i9.1526. doi:10.18103/mra.v5i9.1526

3. Cronje L. Bardet-Biedl syndrome: expect the unexpected, suspect the unsuspected. Southern African Journal of Anaesthesia and Analgesia . 2017;23(5):129-138. doi:10.1080/22201181.2017.1379719

4. Beales PL, Elcioglu N, Woolf AS, Parker D, Flinter FA. New criteria for improved diagnosis of BardetBiedl syndrome: results of a population survey. J Med Genet . 1999;36(6):437-446.

5. Madireddi J, Acharya V, Suryanarayana J, Hande HM, Shetty R. Bardet-Biedl syndrome: multiple fingers with multiple defects!. BMJ Case Rep . 2015;2015:bcr2015211776. Published 2015 Nov 26. doi:10.1136/bcr-2015-211776

6. Forsythe E, Beales P. Bardet-Biedl syndrome. European Journal of Human Genetics . 2012;21(1):8-13. doi:10.1038/ejhg.2012.115 
7. Mahmood S, Khan M, Qadar L, et al. A Unique Manifestation of Bardet-Biedl Syndrome with Otolaryngologic Symptoms and Bronchopneumonia in a One-year-old Girl. Cureus 2019; 11(9): e5717. doi:10.7759/cureus.5717

8. Kalathia M, Parikh Y, Parmar P, Verma S. Rare cause of pediatric obesity: Bardet - Biedl Syndrome. Medical Journal of Dr DY Patil University . 2014;7(2):198. doi:10.4103/0975-2870.126341.

9. Forsythe E, Sparks K, Best $\mathrm{S}$ et al. Risk Factors for Severe Renal Disease in BardetBiedl Syndrome. Journal of the American Society of Nephrology . 2016;28(3):963-970. doi:10.1681/asn.2015091029

10. Forsythe E, Kenny J, Bacchelli C, Beales P. Managing Bardet-Biedl Syndrome - Now and in the Future. Front Pediatr . 2018;6. doi:10.3389/fped.2018.00023

11. O. Khan A, I. Traboulsi E. Bardet-Biedl Syndrome (BBS). American Academy of Ophthalmology . https://www.aao.org/disease-review/bardet-biedl-syndrome-bbs. Published 2016. Accessed November $13,2019$.

12. Flynn JT, Kaelber DC, Baker-Smith CM, et al; SUBCOMMITTEE ON SCREENING AND MANAGEMENT OF HIGH BLOOD PRESSURE IN CHILDREN. Clinical Practice Guideline for Screening and Management of High Blood Pressure in Children and Adolescents.Pediatrics. 2017; 140(3):e20171904. Pediatrics . 2017;140(6):e20173035. doi:10.1542/peds.2017-3035

Number of Tables: 1

Number of figures: 1

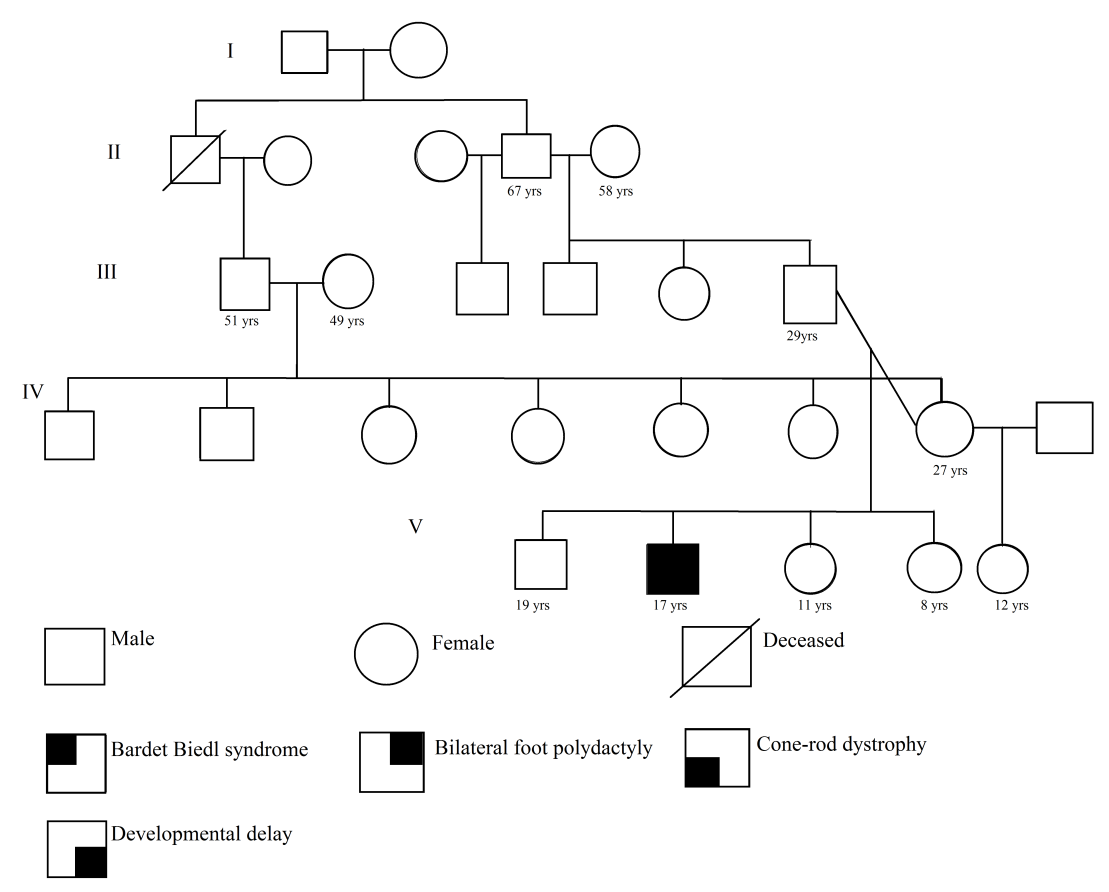

